\title{
Modeling and Its Adaptive Fault-tolerant Control for UAV Two-axis Pan-title
}

\author{
LIN Feng \\ School of automation \\ Shenyang Aerospace University \\ Shenyang, China \\ lfshenyang163@163.com \\ WANG Xiaoxiao \\ School of automation \\ Shenyang Aerospace University \\ Shenyang, China \\ sdqxwxx@163.com
}

\author{
QU Xiaoguang \\ School of automation \\ Shenyang Aerospace University \\ Shenyang, China \\ qxgshenyang163@163.com
}

\begin{abstract}
According to the kinematics and dynamics characteristic of UAV Two-axis Pan-title, the dynamic equations of two-axis platform was erected up, and moreover by predigesting and linearizing the model in the balance point, the math model was gained which could be the same with Fault-tolerant controller design. Applying the adaptive fault-tolerant control method which was real time estimation of actuator faults; the adaptive fault-tolerant controller parameters are updating automatically to compensate the fault effects on systems. The disturbance tolerance ability of the adaptive controller is better than the fixed gain controller's, especially in the actuator saturation and actuator failures. The proposed algorithm was obtained in the form of linear matrix inequalities, and calculating and simulating used the aid of MATLAB LMI Control Toolbox. The results show that the disturbance tolerance ability of this controller is strong, and meeting the needs of stability and security control on UAV two-axis pan-title airborne system.
\end{abstract}

Keywords- Two-axis Pan-title ; Modeling ; linear matrix inequalities ; adaptive control; saturation and failures;

\section{INTRODUCTION}

UAV airborne stabilized pan-tilt system in the low altitude remote sensing technology plays an important role in the system .The system have to eliminate airborne disturbance and actuator failure problems to make high quality of low altitude remote sensing images. There are higher requirements of the fault- tolerance and the antijamming ability. The current modeling and stable control of three-axis tilt has been research more perfect, however, in order to reduce costs and improve efficiency in the use of components, low altitude remote sensing technology mainly uses two-axis table system, and two-axis pan-tilt system have more stringent requirements for stability and fault-tolerance technology.

In order to improve the reliability and safety of the system, using adaptive $\mathrm{H}_{\infty}$ fault-tolerant control algorithm which considers actuator saturation and failures from the point of design .

\section{BRIEF INTRODUCTION OF THE TWO-AXIS PAN-TITLE}

Two-axis pan-tilt has two rotational degrees of freedom, selecting the rectangular coordinate type in the low altitude remote sensing; the structure from the inside to the outside are rolling ring and the pitching loop. The roll frame is connected with the pitch frame. The roll frame can rotate around the pitch frame. The pitch is connected with the base. The base is fixed in UAV.

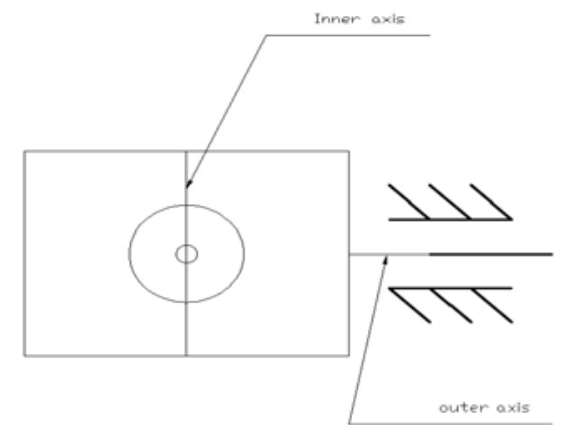

Figure.1 two-axis pan-title frame structure diagram

\section{MODELING OF THE TWO-AXIS PAN-TITLE}

According to the force and space rotation of the frame, we can gain the equation of kinematics and dynamics about the pan-title.

The actual motion of the frame is superimposed fixed-point rotation and horizontal motion in space, including two rotational degrees .The paper did not consider the elastic deformation of the frame, in order to get a simplified mathematical model .

Pan-title mounted on the base of the UAV, the base relative to the inertial coordinate system rotation or translation together with UAV system. The definition of inertial coordinates is $\boldsymbol{O}-\boldsymbol{X a Y a Z a}$; the base is fixedly connected with the moving coordinate system: $\boldsymbol{O}-\boldsymbol{X} \boldsymbol{b} \mathbf{Y} \boldsymbol{b Z \boldsymbol { Z }}$; pitch frame fixed moving coordinate system: $\boldsymbol{O}-\boldsymbol{X p Y p Z p}$; roll is fixed to a frame moving coordinate system: $\boldsymbol{O}$ $\boldsymbol{X r Y r Z r}$; which $\boldsymbol{X p}$ is coincident with $\boldsymbol{X b}$. the relative 
rotation angle which $\boldsymbol{p}$ system and $\boldsymbol{b}$ system is ; the relative rotation angle which $\boldsymbol{r}$ system and $\boldsymbol{p}$ system is $\theta$.

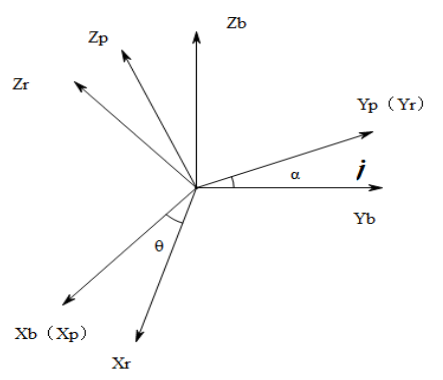

Figure.2 Frame coordinate diagram

The transformation matrix from the base coordinate system to the outer frame coordinate system:

$$
T_{b p}=\left[\begin{array}{ccc}
1 & 0 & 0 \\
0 & \cos \alpha & \sin \alpha \\
0 & -\sin \alpha & \cos \alpha
\end{array}\right]
$$

The transformation matrix the outer frame coordinate system to the inner frame coordinate system:

$$
T_{p r}=\left[\begin{array}{ccc}
\cos \theta & 0 & -\sin \theta \\
0 & 1 & 0 \\
\sin \theta & 0 & \cos \theta
\end{array}\right]
$$

Set the base for the relative angular velocity:

$$
\omega_{\mathrm{b}}=\left[\begin{array}{lll}
p & q & r
\end{array}\right]^{T}
$$

Angular velocity vector of the outer frame itself on the outer coordinate system described as: $\quad \dot{\alpha}=\left[\begin{array}{ccc}\dot{\alpha} & 0 & 0\end{array}\right]^{T}$

The angular velocity of the carrier table change to frame coordinate system as: $\quad \omega_{b p}=T_{b p} \cdot \omega_{b}$

Get on the outer frame coordinate angular velocity vector as follows:

$$
\begin{aligned}
& \omega_{\mathrm{p}}=\omega_{\mathrm{bp}}+\dot{\alpha} \\
& =\left[\begin{array}{lll}
\mathrm{p}+\dot{\alpha} & \mathrm{q} \cos \alpha+\mathrm{r} \sin \alpha & -\mathrm{q} \sin \alpha+\mathrm{r} \cos \alpha
\end{array}\right]^{T} \\
& =\left[\begin{array}{lll}
\omega_{\mathrm{px}} & \omega_{\mathrm{py}} & \omega_{\mathrm{pz}}
\end{array}\right]^{T}
\end{aligned}
$$

Angular velocity vector of the inner frame itself on the inner coordinate system described as: $\dot{\theta}=\left[\begin{array}{ccc}0 & 0 & \dot{\theta}\end{array}\right]^{T}$

Similarly, the inner frame with respect to the inertial system angular velocity:

$$
\begin{aligned}
& \omega_{\mathrm{r}}=\omega_{\mathrm{pr}}+\dot{\theta} \\
& =\left[\begin{array}{c}
\mathrm{p} \cos \theta+\mathrm{q} \cos \alpha \sin \theta+\mathrm{r} \sin \alpha \sin \theta+\dot{\alpha} \cos \theta \\
-\mathrm{p} \sin \theta+\mathrm{q} \cos \alpha \cos \theta+\mathrm{r} \sin \alpha \cos \theta-\dot{\alpha} \sin \theta \\
-\mathrm{q} \sin \alpha+\mathrm{r} \cos \alpha+\dot{\theta}
\end{array}\right] \\
& =\left[\begin{array}{lll}
\omega_{\mathrm{rx}} & \omega_{\mathrm{ry}} & \omega_{\mathrm{rz}}
\end{array}\right]^{T}
\end{aligned}
$$

Because of the frame relative to the shaft is symmetrical, by the theorem of moment $\frac{d \bar{H}}{d \mathrm{t}}=\bar{M}$ get:

The outer frame dynamics equation:

$M_{p}=\left[\begin{array}{lll}M_{p x} & M_{p y} & M_{p z}\end{array}\right]^{T}$

$M_{p x}=J_{p x} \dot{\omega}_{p x}+\left(J_{p z}-J_{p y}\right) \omega_{p z} \omega_{p y}$

$M_{p y}=J_{p y} \dot{\omega}_{p y}+\left(J_{p x}-J_{p z}\right) \omega_{p x} \omega_{p z}$

$M_{p z}=J_{p z} \dot{\omega}_{p z}+\left(J_{p y}-J_{p x}\right) \omega_{p y} \omega_{p x}$

Inner and outer frame are both square. The moment of inertia as follows:
$J_{p x}=J_{p y}=J_{p z}=0.0125 \cdot N \cdot \mathrm{m} / \mathrm{s}^{2} / \mathrm{rad}$

$J_{r x}=J_{r y}=J_{r z}=0.0171 \cdot \mathrm{N} \cdot \mathrm{m} / \mathrm{s}^{2} / \mathrm{rad}$

The inner frame dynamics equation:

$$
\begin{aligned}
& M_{r}=\left[\begin{array}{lll}
M_{r x} & M_{r y} & M_{r z}
\end{array}\right]^{T} \\
& M_{r x}=J_{r x}\left(-\dot{\theta} \omega_{p x} \sin \theta+\dot{\omega}_{p x} \cos \theta-\dot{\theta} \omega_{p z} \cos \theta-\dot{\omega}_{p z} \sin \theta\right) \\
& M_{r y}=J_{r y}\left(\dot{\omega}_{p y}+\ddot{\theta}\right) \\
& M_{r z}=J_{r z}\left(\dot{\theta} \omega_{p x} \cos \theta+\dot{\omega}_{p x} \sin \theta-\dot{\theta} \omega_{p z} \sin \theta-\dot{\omega}_{p z} \cos \theta\right)
\end{aligned}
$$

The torque expressions of internal and external frame:

$M_{\mathrm{in}}=J_{r y} \ddot{\theta}+J_{r y} \dot{\alpha}(r \cdot \cos \alpha-q \cdot \sin \alpha)+J_{r y} \dot{q} \cdot \cos \alpha+J_{r y} \dot{r} \cdot \sin \alpha$

$M_{\text {out }}=\left(J_{r z}+J_{p x}\right) \ddot{\alpha}-J_{r z} \dot{\theta}(\cos \alpha \cdot p-q \cdot \sin \alpha)+\left(J_{p x}+J_{r z}\right) \dot{p}$

Because of the above mathematical model is too complex and non-linear, not easy to design and Simulation of the controller. Simplification and linearization needs to be done.

Written in matrix form:

$$
\left[\begin{array}{c}
\ddot{\theta} \\
\ddot{\alpha}
\end{array}\right]=A_{1}^{-1} M-A_{1}^{-1} B\left[\begin{array}{c}
\dot{\theta} \\
\dot{\alpha}
\end{array}\right]-A_{1}^{-1} C
$$

When Two-axes frame stability, the balance point: $\alpha=\theta=0, \dot{\alpha}=\dot{\theta}=0$

The definition of state variables: $X=\left[\begin{array}{cccc}\alpha & \theta & \dot{\alpha} & \dot{\theta}\end{array}\right]^{T}$ Gain the Equation of state:

$$
\begin{aligned}
& \dot{X}=A X+B_{2} u+B_{1} \omega \\
& Y=C_{2} X=\left[\begin{array}{ll}
\dot{\theta} & \dot{\alpha}
\end{array}\right]^{T}
\end{aligned}
$$

Among them,

$$
\begin{aligned}
u & =\left[\begin{array}{ll}
M_{\mathrm{in}} & M_{\mathrm{out}}
\end{array}\right]^{T}, \omega=\left[\begin{array}{lll}
\dot{p} & \dot{q} & \dot{r}
\end{array}\right]^{T}, \\
A & =\left[\begin{array}{cccc}
0 & 0 & 1 & 0 \\
0 & 0 & 0 & 1 \\
0 & 0 & 0 & -r \\
0 & 0 & \frac{p \cdot J_{r z}}{J_{r z}+J_{p x}} & 0
\end{array}\right], B_{2}=\left[\begin{array}{cc}
0 & 0 \\
0 & 0 \\
\frac{1}{J_{r y}} & 0 \\
0 & \frac{1}{J_{r z}+J_{p x}}
\end{array}\right],
\end{aligned}
$$$$
B_{1}=\left[\begin{array}{ccc}
0 & 0 & 0 \\
0 & 0 & 0 \\
0 & -1 & 0 \\
-1 & 0 & 0
\end{array}\right], C_{2}=\left[\begin{array}{llll}
0 & 0 & 1 & 0 \\
0 & 0 & 0 & 1
\end{array}\right]
$$

For a two-axis pan-tilt system: $p=0.0875 \mathrm{rad} / \mathrm{s}, r=0.0525 \mathrm{rad} / \mathrm{s}$;

After calculation, we obtained state matrixs:

$$
A=\left[\begin{array}{cccc}
0 & 0 & 1 & 0 \\
0 & 0 & 0 & 1 \\
0 & 0 & 0 & -r \\
0 & 0 & \frac{p \cdot J_{r z}}{J_{r z}+J_{p x}} & 0
\end{array}\right]=\left[\begin{array}{cccc}
0 & 0 & 1 & 0 \\
0 & 0 & 0 & 1 \\
0 & 0 & 0 & -0.0525 \\
0 & 0 & 0.0505 & 0
\end{array}\right]
$$




$$
B_{2}=\left[\begin{array}{cc}
0 & 0 \\
0 & 0 \\
\frac{1}{J_{r y}} & 0 \\
0 & \frac{1}{J_{r z}+J_{p x}}
\end{array}\right]=\left[\begin{array}{cc}
0 & 0 \\
0 & 0 \\
58.4795 & 0 \\
0 & 33.7838
\end{array}\right]
$$

\section{ADAPTIVE FAULT-TOLERANT CONTROLLER DESIGN}

UAV pan-title stability frame system is the key to obtain high quality low altitude remote sensing images. The physical characteristics of the frame and servo motor can produce a variable range which is a actuator saturation phenomenon. When the attitude of the UAV changes, the system will produce certain disturbance and fault. So the pan-tilt system have higher demanding requirements on the stability of fault-tolerant control technology.

The state equation of the two-axis pan-title frame system is transformed the general equations of state.

$$
\begin{aligned}
& \dot{x}(t)=A x(t)+B_{1} w(t)+B_{2} \sigma(u) \\
& Z(t)=C x(t)+D \sigma(u)
\end{aligned}
$$

Among them, $x(t)$ is state of the pan-tilt stability system, $\sigma(u) \in R^{m}$ is the input of the PTZ frame system with saturation phenomenon, $\omega(t)$ is generated by the system disturbance input $L_{2}[0, \infty]$. The pan-tilt system (1) of the coefficient matrix corresponding to in the parameter matrix (2).

For the two-axis table system, when the input of the system reaches the saturated situation, the large disturbance may cause the system state and the output divergence and the system work abnormally. So, this paper assumes that the system is bounded external disturbance. The system only make the pan-tilt stability system work normally in practical work if the bounded external disturbances.

Two-axis table system can be transformed into the system with actuator failures and actuator saturation phenomenon:

$$
\begin{aligned}
& \dot{x}(t)=A x(t)+B_{1} \omega+B_{2}(1-\rho) \sigma(u(t)) \\
& z(t)=C x(t)+D(1-\rho) \sigma(u(t))
\end{aligned}
$$

The controller can be set to a structure with adaptive parameter, as follows:

$$
\begin{aligned}
& K_{a}(\hat{\rho}(t))=\sum_{j=1}^{m} K_{a j} \hat{\rho}_{j}(t) \\
& K_{b}(\hat{\rho}(t))=\sum_{j=1}^{m} K_{b j} \hat{\rho}_{j}(t)
\end{aligned}
$$$$
u(t)=K(\hat{\rho}(t)) x(t)=\left(K_{0}+K_{a}(\hat{\rho}(t))+K_{b}(\hat{\rho}(t))\right) x(t)
$$$$
\hat{\rho}(t) \text { is a estimated value for } \rho \text {; In that, }
$$

Description: Through (3) shows, compared with $u(t)=K_{0} x(t)$ in the fixed gain controller, structure of the system is added $K_{a}(\hat{\rho}(t))$ and $K_{b}(\hat{\rho}(t))$ which is the function of $\hat{\rho}$. Because $\hat{\rho}$ is the estimated value, and the system can real-time update the value of the $\hat{\rho}$. By realtime estimating the $\rho$, the gain of the controller can be adjusted online. In this way, so that better performance of control system.
The marking variables $\eta_{i}$ is the function which is composed of $x$ and $\hat{\rho}$. The values of the parameters can be real-time measured. $\eta_{i}$ is the response of the actuator saturation. Under the same conditions, $\eta_{i}$ have the different expression. In the reference [3] gives details of the method.

The adaptive laws $\dot{\hat{\rho}}_{j}(t)$ in the system can be adjusted online:

$$
\begin{gathered}
\dot{\hat{\rho}}_{j}=\operatorname{Pr} o j_{\left[\min _{q}\left\{\rho_{-}^{q}\right\} \max _{q}\left\{\bar{\rho}_{j}^{q}\right\}\right]}\left\{L_{1 j}\right\} \\
=\left\{\begin{array}{cc} 
& \hat{\rho}_{j}=\min _{q}\left\{\underline{\rho}_{j}^{q}\right\} \& \mathrm{~L}_{1 i} \leq 0 \\
0 & \hat{\rho}_{j}=\max _{j}\left\{\bar{\rho}_{j}^{q}\right\} \& \mathrm{~L}_{1 \mathrm{j}} \leq 0 \\
L_{1 j} & \text { other }
\end{array}\right.
\end{gathered}
$$

$\mathrm{L}_{1 \mathrm{i}}=-l_{i} x^{T}(t)\left[P B_{2}\left(\sum_{i=0}^{2^{m}-1} \eta_{i} D_{i}\right) K_{a j}+P B_{2}^{j}\left(\sum_{i=0}^{2^{m}-1} \eta_{i} D_{i}\right) K_{b}(\hat{\rho})\right.$

$P=\delta X^{-1}, K_{0}=Y_{0} X^{-1}, K_{a j}=Y_{a j} X^{-1}, K_{b j}=Y_{b j} X^{-1}$, $H_{a j}=O_{a j} X^{-1} \quad, \quad H_{b j}=O_{b j} X^{-1}$.Among them, $l_{j}>0(j \in I[1, m])$ is the adaptive gain law. The gain structure of system controller:

$$
K(\hat{\rho})=K_{0}+\sum_{j=1}^{m} \hat{\rho}_{j} K_{a j}+\sum_{j=1}^{m} \hat{\rho}_{j} K_{b j}
$$

Note: $\gamma_{n}$ is adaptive $H_{\infty}$ performance index on the troublefree condition. $\gamma_{f}$ is adaptive $H_{\infty}$ performance index on the trouble condition. $\delta$ is the disturbance tolerance level of the system.

Through the optimization algorithm to get the minimization $\gamma_{f}$ and $\gamma_{n}$, the maximum $\delta$ :

$$
\min \eta=\alpha \eta_{n}+\beta \eta_{f}+\gamma \eta_{\delta} \quad, \quad \text { s.t. }(a) \quad,
$$

$\varepsilon^{*}\left(P, \delta^{*}\right) \subset \wp(H(\hat{\rho})) \quad, \quad \eta_{n}=\gamma_{n}^{2} \quad, \quad \eta_{f}=\gamma_{f}^{2} \quad$ and

$$
\eta_{\delta}=\frac{1}{\delta^{*}}=\frac{1}{\delta+\max \left\{\sum_{j=1}^{m} \frac{\bar{\rho}_{j}^{2}(t)}{l_{j}}\right\}}
$$

Among them, $\alpha, \beta, \gamma$ is the weighted coefficient.

\section{EXPERIMENT AND CONCLUSIONS}

There are two modes of actuator pan-title stabilization system: normal mode and fault mode.

Normal mode: the pitch and roll box frame motor is in normal state, i.e. $\rho_{1}^{1}=\rho_{2}^{1}=0$;

Failure mode: one motor is in failure, i.e. $\rho_{1}^{2}=1,0 \leq \rho_{2}^{2} \leq a ; a=0.8$,

Among them , $a=0.8$ is the biggest failure rate of actuator.

Set the weighting parameter $\alpha=0.5$, $\beta=0.01, \gamma=0.5$. Calling the Matlab of the LMI toolbox to solve this problem. As the fixed gain controller design method $\eta_{n}=16.3668, \eta_{f}=490.4561$, 
$\eta_{\delta}=26.1502$ is obtained; and, as the adaptive control algorithm obtained $\eta_{n}=11.3668, \quad \eta_{f}=100.6399$, $\eta_{\delta}=1.4438$; As is shown above, the adaptive faultcontroller is better than the fixed gain controller.

Considering the problem of disturbance tolerance, in the following simulation selection disturbance:

$$
\omega(t)=\left\{\begin{array}{cc}
200, & 4 \leq t \leq 5 \\
0 & \text { other }
\end{array}\right.
$$

Fig .3 shows the state curve of the closed-loop system in the adaptive controller of normal model. Fig .4 shows the state curve of the closed-loop system with fixed gain controller in normal mode. Obviously, on the disturbance (6), in the role of the adaptive controller for the closed loop system remains asymptotically stable, but in the fixed gain controller is divergent. This phenomenon indicates that the adaptive method in this paper has better effect.

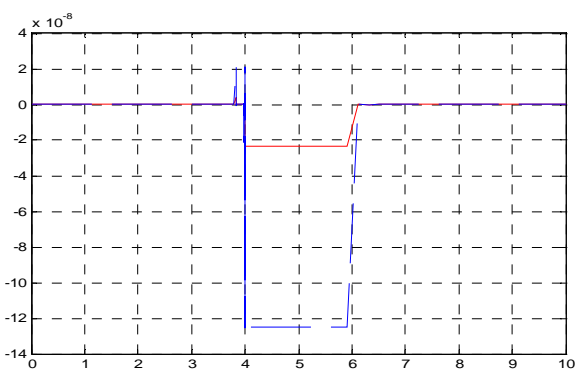

Figure. 3 Trajectories of closed-loop systems with adaptive controller in normal case

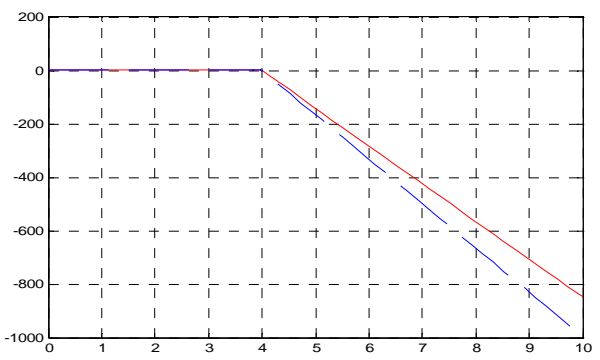

Figure. 4 Trajectories of closed-loop systems with fixed gains controller in normal case

\section{REFERENCES}

[1] Maybeck P S. Multiple adaptive algorithm for detecting and compensating sensor and actuator/surface failures in aircraft flight control systems[J], International Journal of Robust and Nonlinear Control, 1999, 9: 1051-1070.

[2] Liu S Z, Sun L H, Che H Modeling and Its Hळ Stabilization Control for Airborne Electro-optical Tracking and Pointing Platform [A] Northwestern Polytechnical University, 2008,20(6):1518-1521 (in Chinese).

[3] Guan W by Stability Analysis and Fault-tolerant Control of Constrained Systems Based on LMI Technique Northeastern University ,2008 (in Chinese).

[4] Zhang P modeling and control of airborne/missile-borne visionguidance stabilized platfo [M].1rd National Defence Industry Press,2011-6-1(in Chinese).

[5] Zeng Q S ,Wang M , Liu S C ,The Study Dynamics Coupling and Decoupling between Frames of Three Axis Turn -table Journal of Chinese inertial technology 1997, 5(3) 44-49.( in Chinese)

[6] Shimemura E, Fujita M. A design method for linear state feedback systems possessing integrity based on a solution of a Riccati-type equation[J], Int. J. Control,1985, 42(4): 887-899.

[7] Yang G H, Wang J L, Yeng C S. Reliable guaranteed cost control for uncertain nonlinear systems [J], IEEE Trans. Automatic Control., 2000, 45(11): 2188-2192

[8] Xin Z K ,Fang Y C,Zhang X B Adaptive control for the on-board pan-tilt camera in an UAV ground-target-tracking system $[\mathrm{J}]$ Control Theory \& Applications 2010,27(8):1001-1006 ( in Chinese)

[9] Meng G..The Design of UAV Visual Stabilization Platform System[D] Harbin Engineering University ,2011(in Chinese)

[10] Jiang J. Design of reconfigurable control systems using eigenstructure assignments[J], International Journal of Control, 1994, 59(2): 394-410 